\title{
Respuesta superovulatoria y embrionaria en alpacas según el número de folículos reclutados por onda folicular
}

\section{Superovulatory and embryonic response in alpacas according to the number of follicles recruited by follicular wave}

\author{
Alfredo Pozo C. ${ }^{1,4}$, Álvaro Vásquez Y. ${ }^{1}$, Juan Zevallos A. ${ }^{2}$, Luis Olivera M. ${ }^{2}$, \\ Aída Cordero R. ${ }^{3}$, Wilfredo Huanca L. ${ }^{1,5}$
}

\section{RESUMEN}

\begin{abstract}
El propósito del estudio fue evaluar la respuesta superovulatoria y embrionaria en alpacas con gonadotropina coriónica equina (eCG) según el número de folículos reclutados $\geq 3 \mathrm{~mm}$ en la onda folicular (OF). Se seleccionaron 26 alpacas del CIP «Chuquibambilla», Universidad Nacional del Altiplano, Puno, Perú, para conformar dos grupos experimentales: GE01 ( $\mathrm{n}=15$ alpacas) con menos de tres folículos $\geq 3 \mathrm{~mm}$ y GE02 ( $\mathrm{n}=11$ alpacas) con más de cuatro folículos $\geq 3 \mathrm{~mm}$ de diámetro al reclutamiento folicular pos-inducción de la ovulación de un folículo $\geq 7 \mathrm{~mm}$ con $0.0042 \mathrm{mg}$ de acetato de buserelina (AB). Ambos grupos fueron evaluados por ecografía cada dos días hasta inicios de la tercera onda folicular. Posteriormente, hembras con folículos $\geq 7 \mathrm{~mm}$ fueron inducidas a la ovulación con $\mathrm{AB}$ (día 0 ), se verificó la ovulación a las 36 h y se administró 700 UI de eCG. Al día 6.5 se hizo el empadre y 7.5 días después se colectaron los embriones. La respuesta superovulatoria fue similar en GE01 y GE02 con $4.4 \pm 4.0$ y $4.7 \pm 3.7$ folículos entre $\geq 3$ y $<7$ $\mathrm{mm} ; 7.5 \pm 3.7$ y $10.1 \pm 6.3$ folículos $\geq 7 \mathrm{~mm} ; \mathrm{y} 7.2 \pm 3.3$ y $8.6 \pm 5.0$ cuerpos lúteos, respectivamente. Además, GE01 y GE02 presentaron $1.6 \pm 0.5$ y $2.8 \pm 1.3$ folículos anovulatorios luteinizados y $2.0 \pm 1.0$ y $2.0 \pm 2.2$ folículos anovulatorios, respectivamente. En $80 \%$ de alpacas en GE01 se colectaron $2.5 \pm 1.4$ embriones y en $70 \%$ de alpacas de GE02 se colectaron $2.3 \pm 1.1$ embriones. Así mismo, se colectaron 25 embriones transferibles y 5 no transferibles en GE01 ( $\mathrm{n}=15)$ y 12 embriones transferibles y 4 no transferibles en GE02
\end{abstract}

${ }^{1}$ Laboratorio de Reproducción Animal, Facultad de Medicina Veterinaria, Universidad Nacional Mayor de San Marcos, Lima, Perú

${ }^{2}$ Facultad de Medicina Veterinaria y Zootecnia, Universidad Nacional del Altiplano, Puno, Perú

${ }^{3}$ Departamento de Nutrición, Facultad de Zootecnia, Universidad Nacional Agraria, La Molina, Lima, Perú

${ }^{4}$ E-mail: alfredo.pozo@unsch.edu.pe

${ }^{5}$ E-mail: whuancal@unmsm.edu.pe

Recibido: 26 de octubre de 2020

Aceptado para publicación: 13 de marzo de 2021

Publicado: 24 de abril de 2021 
$(\mathrm{n}=10)$. Los resultados sugieren que no hay diferencia en la respuesta ovárica y colección embrionaria en alpacas superestimuladas con eCG a pesar de una marcada diferencia de folículos emergentes $\geq 3 \mathrm{~mm}$ en la primera OF y que no se hizo sostenible en la segunda y tercera OF.

Palabras clave: alpaca, eCG, fertilidad, onda folicular, respuesta ovárica, superovulación

\section{Abstract}

The aim of this study was to evaluate the superovulatory and embryonic response in alpacas with equine chorionic gonadotropin (eCG) according to the number of follicles recruited $\geq 3 \mathrm{~mm}$ in the follicular wave (FW). Twenty-six alpacas from the CIP «Chuquibambilla», Universidad Nacional del Altiplano, Puno, Peru, were selected and distributed into two experimental groups: GE01 ( $\mathrm{n}=15$ alpacas) with less than three follicles $\geq 3 \mathrm{~mm}$ and GE02 ( $\mathrm{n}=11$ alpacas) with more from four follicles $\geq 3 \mathrm{~mm}$ in diameter to follicular recruitment post-induction of ovulation by a follicle $\geq 7 \mathrm{~mm}$ with $0.0042 \mathrm{mg}$ of buserelin acetate (BA). Both groups were evaluated by ultrasound every other day until the beginning of the third follicular wave. Subsequently, females with follicles $\geq 7 \mathrm{~mm}$ were induced to ovulate with BA (day 0), ovulation was verified at $36 \mathrm{~h}$ and $700 \mathrm{IU}$ of eCG was administered. On day 6.5 animals were mated and 7.5 days later the embryos were collected. The superovulatory response was similar in GE01 and GE02 with $4.4 \pm 4.0$ and $4.7 \pm 3.7$ follicles between $\geq 3$ and $<7 \mathrm{~mm} ; 7.5 \pm 3.7$ and $10.1 \pm 6.3$ follicles $\geq 7 \mathrm{~mm}$; and $7.2 \pm 3.3$ and $8.6 \pm 5.0$ corpora lutea, respectively. Furthermore, GE01 and GE02 presented $1.6 \pm 0.5$ and $2.8 \pm 1.3$ luteinized anovulatory follicles and $2.0 \pm 1.0$ and $2.0 \pm 2.2$ anovulatory follicles, respectively. In $80 \%$ of alpacas in GE01 $2.5 \pm 1.4$ embryos were collected and in $70 \%$ of alpacas in GE02 $2.3 \pm 1.1$ embryos were collected. Likewise, 25 transferable and 5 nontransferable embryos were recovered in GE01 $(n=15)$ and 12 transferable and 4 nontransferable embryos in GE02 $(n=10)$. The results suggest that there is no difference in the ovarian response and embryonic collection in alpacas overstimulated with eCG despite a marked difference in emerging follicles $\geq 3 \mathrm{~mm}$ in the first FW and that it was not sustainable in the second and third FW.

Key words: alpaca, eCG, fertility, follicular wave, ovarian response, superovulation

\section{INTRODUCCIÓN}

La alpaca constituye un valioso recurso para el poblador altoandino, porque le provee carne y fibra de alta calidad. El interés por la masificación de genes de alto valor productivo en la alpaca ha favorecido la introducción y desarrollo de tecnologías reproductivas asistidas (TRA), como la ovulación múltiple, producción in vivo e in vitro de embriones y la trasferencia de embriones a hembras recipientes.

La ovulación múltiple y producción de embriones en alpacas ha utilizado diversos protocolos basados en la FSH y eCG solas o en combinación. Dosis de 400 UI durante 3 días y por vía $\mathrm{SC}$ en presencia de un folículo $<7 \mathrm{~mm}$ (Novoa y Sumar, 1968), 650 UI en dosis única por vía IM durante el inicio de la onda folicular (Cervantes, 2008) o 1000 UI 
de eCG (Vivanco et al., 2014b), produjeron variadas respuestas ováricas en el número de folículos, cuerpos lúteos (CLs), e incluso en la formación de folículos anovulatorios y luteinizados con efectos directos sobre la recuperación embrionaria (Tibary et al., 2015). Dicha variabilidad ha sido asociada a factores intra y extraováricos (Webb y Campbell, 2007), haciendo que la técnica genere desconfianza por demandar gastos, esfuerzos y tiempo. De otro lado, esta variabilidad ha motivado a profundizar el conocimiento de los procesos fisiológicos que controlan el desarrollo folicular ovárico con la finalidad de desarrollar estrategias e incrementar la eficiencia reproductiva (Rosales et al., 2012).

La aplicación de la ecografía como método poco invasivo en el campo de la reproducción animal ha facilitado la comprensión en tiempo real de procesos y eventos reproductivos poco conocidos. Por ejemplo, el inicio de la onda folicular en la alpaca fue caracterizada por la aparición de varios folículos (8-10) con diámetros $<3 \mathrm{~mm}$, seguido por el crecimiento continuo de, por lo general, uno, a veces dos o tres folículos de 3-5 $\mathrm{mm}$, los cuales siguen creciendo o mantienen su tamaño durante los primeros ocho días después de la emergencia (Vaughan et al., 2004). De igual forma, el inicio de la onda folicular en alpacas ha sido estimada en 4.3 \pm 1.4 días (Andrade, 2007) y con $2.8 \pm 1.3$ (rango de 1-8) de folículos emergentes $\geq 3$ $\mathrm{mm}$ y repetibles $(\mathrm{r}=0.72)$ en ondas foliculares sucesivas (Vásquez, 2018).

El recuento de folículos antrales (RFA) en bovinos ha tomado importancia en las últimas décadas. Se han cuantificado ecográficamente los folículos antrales $\geq 3 \mathrm{~mm}$ con una amplia variabilidad entre animales ( 5 a 50 folículos) y con alta repetibilidad ( $\mathrm{r}=0.89)$ (Ireland et al., 2007; Evans et al., 2011). El RFA apoya la clasificación fenotípica de vacas con mayor o menor número de folículos, reflejado con la mayor o menor reserva ovárica, la fertilidad, concentración de hormona antimülleriana (Ireland et al., 2008), progesterona y espesor endometrial (Jimenez-Krassel et al., 2009). En este contexto, la respuesta ovárica a la superestimulación pudo predecirse ecográficamente por el número de folículos $\geq 2 \mathrm{~mm}$ a la aparición de las ondas foliculares en vacas (Singh et al., 2004). Por lo tanto, el presente estudio tuvo la finalidad de evaluar la respuesta superovulatoria a la eCG en alpacas según el número intrínseco de folículos emergentes en la onda folicular.

\section{Materiales y Métodos}

\section{Lugar de Ejecución}

El estudio se desarrolló en el CIP «Chuquibambilla» de la Universidad Nacional del Altiplano (UNA), departamento de Puno, Perú, a 3974 msnm, entre enero y abril de 2016.

\section{Animales}

Se utilizaron 26 alpacas seleccionadas de un total de 34 alpacas hembra Suri. Los animales tenían entre 4 y 7 años, 2.5 a 3.5 de condición corporal (escala de 1 a 5) (Van Saun y Herdt, 2014), $69.2 \pm 7.5 \mathrm{~kg}$ de peso corporal, con un parto como mínimo. Asimismo, se encontraban vacías y sin problema aparente de tipo reproductivo. Además, se dispuso de 10 alpacas macho Suri para el empadre, los cuales tenían entre 4 y 8 años, condición corporal de $2.75 \mathrm{a} 4,75.8 \pm 8.0 \mathrm{~kg}$ de peso y fertilidad comprobada.

Todos los animales estaban debidamente identificados y fueron alimentados con pastura natural y en ocasiones con heno de avena. El manejo fue similar para todos los animales durante el experimento. 


\section{Diseño Experimental}

Al inicio, se hizo una ecografia transrectal a las 34 hembras con un ecógrafo Aloka SSD500 y un transductor lineal rectal de 5.0 $\mathrm{MHz}$ para localizar un folículo ovárico $\geq 7 \mathrm{~mm}$ de diámetro. Ante esto, se indujo la ovulación con $0.0042 \mathrm{mg}$ de acetato de buserelina (AB) (Conceptal ${ }^{\circledR}$, Intervet, $1 \mathrm{ml} \mathrm{IM}$ ) considerando este evento como el día 0 (Huanca et al., 2001). A los dos días se verificó ecográficamente la ovulación. Además, considerando el intervalo de tiempo ( $4.3 \pm 1.4$ días postratamiento con $\mathrm{GnRH}$ ) que demanda el inicio de la onda folicular (Andrade, 2007) y evaluándose retrospectivamente el folículo dominante, se determinó el reclutamiento folicular con la aparición de folículos $\geq 3 \mathrm{~mm}$, siguiendo la metodología descrita en llamas por Adams et al. (1990).

Las 26 alpacas seleccionadas fueron asignadas en dos grupos experimentales, en función al número de folículos emergentes: GE01, alpacas $(\mathrm{n}=15)$ con $\leq 3$ folículos emergentes de $\geq 3 \mathrm{~mm}$ de diámetro, y GE02, alpacas $(n=11)$ con $\geq 4$ folículos emergentes de $\geq 3 \mathrm{~mm}$ de diámetro.

Se realizó la ecografía cada dos días, según la metodología descrita por Bravo et al. (1990) durante dos ondas foliculares e inicios de la tercera, a partir de la cual se inició la superovulación con eCG con el fin de evaluar la respuesta ovárica y embrionaria. Los folículos ováricos fueron medidos mediante ecografía visualizándose como imágenes anecoicas, los CLs como imágenes hipoecogénicas, y los folículos anovulatorios como estructuras anecoicas $>12 \mathrm{~mm}$ de diámetro (Rodriguez et al., 2014) y de paredes delgadas. Asimismo, los folículos anovulatorios luteinizados se observaron como estructuras anecoicas y con las paredes engrosadas hipoecogénicas $\geq 3 \mathrm{~mm}$ y con porciones centrales anecoicas. La tasa de recuperación embrionaria se estimó en función al número de CLs determinados.

\section{Procedimiento Metodológico}

\section{Superovulación}

Los animales de los dos grupos fueron sometidos a un protocolo de superovulación, de acuerdo con lo señalado por Huanca (2005) y Sumar (2013). Se determinó por ultrasonografía la presencia de un folículo preovulatorio $\geq 7 \mathrm{~mm}$ de diámetro en las alpacas de la tercera onda folicular. Se administró un análogo de GnRH $(0.0042 \mathrm{mg}$ acetato de buserelina, Conceptal ${ }^{\circledR}$, Intervet, $1 \mathrm{ml}, \mathrm{IM})$ para inducir ovulación, representando el día cero (D 0). A las $36 \mathrm{~h}$ se hizo la ecografía para verificar la ovulación y se administró eCG en dosis única (700 UI eCG, Novormon ${ }^{\circledR}$ Syntex, $3.5 \mathrm{ml}$, IM) como agente superovulatorio. En el D 6.5 se administró cloprostenol $\left(0.25 \mathrm{mg}\right.$, Lutaprost ${ }^{\circledR} 250$, Agrovet Market, $1 \mathrm{ml}$, IM) para producir la luteólisis del CL originado por la inducción de la ovulación del folículo preovulatorio del D 0 , dosis y tiempo suficiente como lo descrito por Mamani et al. (2016). Al D 7.5 se registró el número y tamaño de los folículos mediante ecografía e inmediatamente después fueron cubiertas con machos fértiles durante 15 minutos como mínimo, previa verificación de una monta correcta. Posterior a la monta se administró $0.0042 \mathrm{mg}$. de acetato de buserelina $\left(\right.$ Conceptal ${ }^{\circledR}$, Intervet, $\left.1 \mathrm{ml}, \mathrm{IM}\right)$ para garantizar la ovulación. Alas $12 \mathrm{~h}$ subsiguientes se realizó una segunda monta para mejorar la fertilización de los ovocitos.

\section{Colección embrionaria}

Al D 14 se realizó una ecografía transrectal de los ovarios para registrar la cantidad y dimensión de los CLs, así como registrar el número de folículos anovulatorios y luteinizados.

El medio de lavado fue preparado en el Laboratorio de Reproducción de la Universidad Nacional Mayor de San Marcos, conteniendo PBS y enriquecido con albúmina sérica y antibióticos. Previo al lavado intrauterino 
se administró un tranquilizante (acepromazina maleato, Promazil ${ }^{\circledR}$, Montana, $0.5 \mathrm{ml}$, IM) y anestesia epidural en base a lidocaína $2 \%(2.5$ $\mathrm{ml})$ en el espacio sacro coccígeo. Se utilizó un catéter Foley $\mathrm{N}^{\circ} 16 \mathrm{Fr}$ de dos vías fijada internamente por un mandril metálico y protegido por una camiseta sanitaria. El ingreso del catéter por el cérvix fue facilitado mediante fijación manual vía rectal. Una vez que el catéter se ubicara en uno de los cuernos uterinos, se infló el balón del catéter con 15 a $20 \mathrm{ml}$ de aire para asegurarlo al cuerno uterino impidiendo el reflujo del medio y embriones durante el lavado. Este procedimiento se repitió para el otro cuerno uterino.

El lavado intrauterino se hizo a través del sistema discontinuo, utilizando una jeringa de $50 \mathrm{ml}$. Se utilizó $120 \mathrm{ml}$ de medio de lavado temperado $\left(37^{\circ} \mathrm{C}\right)$ por cada cuerno y $30 \mathrm{ml}$ para el enjuague del catéter después de haberlo extraído del tracto reproductivo. El medio de lavado recuperado fue colocado en filtros Encom con $70 \mu \mathrm{m}$ de diámetro, luego transferido a placas petri y colocado en una platina térmica para la búsqueda y evaluación de los embriones. Terminada la recuperación embrionaria por lavado intrauterino, se aplicó cloprostenol $(0.25 \mathrm{mg}$, Lutaprost ${ }^{\circledR} 250$, Agrovet Market, $1 \mathrm{ml}$, IM) para inducir la luteólisis de los CLs en las alpacas de los dos grupos experimentales.

\section{Búsqueda y clasificación de embriones}

La búsqueda de embriones en el medio de lavado contenido en las placas petri cuadriculadas conservadas en platina caliente se hizo con un estereomicroscopio Nikon ${ }^{\circledR}$ a $40 \mathrm{X}$ y siguiendo un orden en cada cuadrícula. La clasificación se basó en la propuesta de Tibary et al. (2015) modificada, como calidad de embriones en Grado I (excelente), Grado II (bueno), Grado III (medio o regular) y Grado IV (mala y embriones no transferibles). El presente trabajo consideró embriones transferibles a los de grado I y II y embriones no transferibles a los grados III y IV.

\section{Análisis de datos}

Los datos se presentan en cuadros mediante promedios \pm desviación estándar, valores mínimos y máximos y en porcentajes. Los datos de las variables estudiadas fueron sometidos a la prueba de Shapiro-Wilk para determinar su distribución normal. Los datos fueron analizados con el programa SPSS v. 22.0. Las diferencias se consideraron significativas cuando $\mathrm{p}<0.05$.

El número de folículos reclutados $\geq 3$ mm por grupo en $1 \mathrm{a} 1^{\circ}, 2^{\circ}$ y $3^{\circ}$ onda folicular, número de folículos $\geq 3 \mathrm{~mm} \mathrm{y}<7 \mathrm{~mm}$, número y tamaño de folículos anovulatorios, y número y tamaño de folículos anovulatorios luteinizados fueron comparados mediante la prueba de hipótesis no paramétrica de MannWhitney. El tamaño de folículos $\geq 7 \mathrm{~mm}$ inducidos a la ovulación al $\mathrm{D} 0$, número de folículos $\geq 3 \mathrm{~mm}$ al D 1.5 (36h), número y tamaño de folículos $\geq 7 \mathrm{~mm}$ al D 7.5, así como, el número y tamaño de CLs y el total de embriones al D 14 del programa superovulatorio, fueron comparados entre grupos mediante la prueba de hipótesis paramétrica t de Student para muestras independientes. La tasa de recuperación embrionaria, así como el total de embriones transferibles, no transferibles y la tasa de embriones colectados según grado de desarrollo (blastocistos, mórulas y ovocitos sin fertilizar) entre grupos fueron comparados mediante la prueba exacta de Fisher.

\section{Resultados y Discusión}

\section{Respuesta Ovárica}

En el Cuadro 1 se muestra que el tamaño de los folículos preovulatorios $\geq 7 \mathrm{~mm}$ inducidos a la ovulación al D 0 no difirieron entre grupos, dado que todas las alpacas llegaron a ovular. Asimismo, no hubo diferencia entre grupos para folículos $<3 \mathrm{~mm}$ al día 1.5 (36h) ni en folículos $\geq 3$ y $<\underline{7} \mathrm{~mm}$. Los folículos 
Cuadro 1. Respuesta ovárica a la superovulación con eCG en alpacas, según los grupos experimentales ${ }^{1}$

\begin{tabular}{|c|c|c|c|c|c|}
\hline \multirow{2}{*}{\multicolumn{2}{|c|}{$\begin{array}{l}\text { Respuesta ovárica al tratamiento } \\
\text { superovulatorio con eCG }\end{array}$}} & \multicolumn{2}{|r|}{ GE01 } & \multicolumn{2}{|r|}{ GE02 } \\
\hline & & \multirow{2}{*}{$\begin{array}{c}\begin{array}{c}\mathrm{N}^{\circ} \\
\text { alpacas }\end{array} \\
15\end{array}$} & \multirow{2}{*}{$\frac{\text { Media } \pm \text { DE }}{9.3^{\mathrm{a}} \pm 2.2}$} & \multirow{2}{*}{$\begin{array}{c}\begin{array}{c}\mathrm{N}^{\circ} \\
\text { alpacas }\end{array} \\
11\end{array}$} & \multirow{2}{*}{$\frac{\text { Media } \pm \mathrm{DE}}{8.6^{\mathrm{a}} \pm 1.1}$} \\
\hline Día 0 & $\begin{array}{l}\text { Diámetro de folículo } \\
\text { preovulatorio (mm) }\end{array}$ & & & & \\
\hline $\begin{array}{l}\text { Día } 1.5 \\
\text { eCG }\end{array}$ & Folículos $\geq 3 \mathrm{~mm}(\mathrm{n})$ & 13 & $\begin{array}{l}2.7^{\mathrm{a}} \pm 0.8 \\
\quad(35)\end{array}$ & 09 & $\begin{array}{l}2.6^{\mathrm{a}} \pm 1.3 \\
\quad(23)\end{array}$ \\
\hline \multirow[t]{3}{*}{$\begin{array}{l}\text { Día } 7.5 \\
\text { empadre }\end{array}$} & $\begin{array}{l}\text { Folículos } \geq 3 \mathrm{~mm} \text { y }<7 \\
\mathrm{~mm}^{2}, \text { Cantidad }(\mathrm{n})\end{array}$ & 14 & $\begin{array}{l}4.4^{\mathrm{a}} \pm 4.0 \\
\quad(61)\end{array}$ & 09 & $\begin{array}{l}4.7^{\mathrm{a}} \pm 3.7 \\
\quad(42)\end{array}$ \\
\hline & $\begin{array}{l}\text { Folículos } \geq 7 \mathrm{~mm} \\
\text { Cantidad (n) }\end{array}$ & 15 & $\begin{array}{l}7.5^{\mathrm{a}} \pm 3.7 \\
\quad(112)\end{array}$ & 11 & $\begin{array}{l}10.1^{\mathrm{a}} \pm 6.3 \\
\quad(111)\end{array}$ \\
\hline & Tamaño (mm) & 15 & $8.4^{\mathrm{a}} \pm 1.1$ & 11 & $8.8^{\mathrm{a}} \pm 0.9$ \\
\hline \multirow{6}{*}{$\begin{array}{l}\text { Día } 14 \\
\text { recuperación } \\
\text { embrionaria }\end{array}$} & $\begin{array}{l}\text { Cuerpos lúteos } \\
\text { Cantidad (n) }\end{array}$ & 15 & $\begin{array}{l}7.2^{\mathrm{a}} \pm 3.3 \\
(108)\end{array}$ & 11 & $\begin{array}{l}8.6^{\mathrm{a}} \pm 5.0 \\
\quad(102)\end{array}$ \\
\hline & Diámetro (mm) & 15 & $10.7^{\mathrm{a}} \pm 1.2$ & 11 & $11.4^{\mathrm{a}} \pm 2.5$ \\
\hline & $\begin{array}{l}\text { Fol. anovulatorios } \\
\text { luteinizados } \geq 11 \mathrm{~mm}^{2} \\
\text { Cantidad }(\mathrm{n})\end{array}$ & 5 & $\begin{array}{l}1.6^{\mathrm{a}} \pm 0.5 \\
\quad(8)\end{array}$ & 4 & $\begin{array}{l}2.8^{\mathrm{a}} \pm 1.3 \\
\quad(14)\end{array}$ \\
\hline & Tamaño (mm) & 5 & $14.5^{\mathrm{a}} \pm 6.3$ & 4 & $16.3^{a} \pm 2.7$ \\
\hline & $\begin{array}{l}\text { Fol. anovulatorios } \geq 12 \\
\mathrm{~mm}^{2} \text {, Cantidad (n) }\end{array}$ & 7 & $\begin{array}{l}2.0^{\mathrm{a}} \pm 1.0 \\
\quad(14)\end{array}$ & 7 & $\begin{array}{l}2.0^{\mathrm{a}} \pm 2.2 \\
\quad(14)\end{array}$ \\
\hline & Tamaño (mm) & 7 & $16.4^{\mathrm{a}} \pm 5.8$ & 7 & $18.4^{\mathrm{a}} \pm 4.2$ \\
\hline
\end{tabular}

$\geq 7 \mathrm{~mm}$ fueron igualmente similares entre grupos, aunque presentaron disímiles coeficientes de variación $(\mathrm{GE} 01=49.3 \%$; GE02 $=$ $62.4 \%)$.

El número y tamaño de los CLs previos a la recuperación intrauterina de embriones el día 14 fueron similares entre grupos (Cuadro 1). Asimismo, las alpacas de ambos gru- pos tuvieron similar predisposición a formar folículos anovulatorios luteinizados y folículos anovulatorios.

La respuesta superovulatoria a la eCG en alpacas en función al promedio de folículos preovulatorios $(\geq 7 \mathrm{~mm})$ y cuerpos lúteos, fueron similares a GE02 (10.1 \pm 6.3 folículos y $8.6 \pm 5.0 \mathrm{CLs})$ y ligeramente superiores a 
GE01 (7.5 \pm 3.7 folículos y 7.2 \pm 3.3 CLs $)$ en comparación a los resultados obtenidos por Mendoza (2012) (10.2 \pm 4.47 folículos y 8.0 $\pm 3.42 \mathrm{CLs}$ ) usando $700 \mathrm{UI}$ de eCG, así como también a los resultados de Huanca (2008) ( $9.74 \pm 1.27$ folículos de 7 a $13 \mathrm{~mm}$ y $9.92 \pm$ 0.99 CLs) usando 750 UI de eCG.

La diferencia significativa en el número de folículos $\geq 3 \mathrm{~mm}$ al inicio de la primera onda folicular (GE01: $\leq 3$ folículos, GE02: $\geq 4$ folículos) (Cuadro 2) tuvo similar número de folículos antrales (folículos entre $\geq 3 \mathrm{~mm}$ y $<7$ $\mathrm{mm}$ y folículos preovulatorios $\geq 7 \mathrm{~mm}$ ) al día 7.5 como respuesta a la aplicación de la eCG, contrastando con los hallazgos de Singh et al. (2004), quienes encontraron una mayor respuesta gonadotrópica (FSH) de mayor número de folículos antrales en vacas con mayor número de folículos $\geq 2 \mathrm{~mm}$ a la aparición de las ondas. El estrecho rango en el número de folículos $\geq 3 \mathrm{~mm}$ a la emergencia de la primera onda folicular (entre 2 a 9 folículos) afectó directamente en la conformación de los GEs e imposibilitaron la agrupación de las alpacas en función al número extremo de folículos emergentes (Ej. 10\% superior y $10 \%$ inferior) tal como Singh et al. (2004) lo hicieran para predecir significativamente la respuesta superestimulatoria. Así mismo, a pesar de que el número de folículos emergentes antrales al inicio de la onda folicular varíe entre ondas consecutivas, como ocurrió en el presente estudio donde los grupos GE01 y GE02 mostraron diferencia significativa en la primera onda folicular, estos fueron similares en la $2^{\text {da }} y 3^{\text {ra }}$ onda folicular. Por lo tanto, es probable que en alpacas existan otros factores no conocidos que no permiten una predicción más certera y que el número de folículos $>3 \mathrm{~mm}$ (GE01: $\leq 3$ folículos, GE02: $\geq 4$ folículos) parece no ser el único factor por considerar.

El inicio de la onda folicular en alpacas tras la inducción de la ovulación de folículos $\geq 7 \mathrm{~mm}$ con $\mathrm{GnRH}$ fue estimada por Andrade (2007) en $4.5 \pm 1.4$ días (rango 2-8). Así mismo, Vásquez (2018) cuantificó el número promedio de folículos $\geq 3 \mathrm{~mm}$ a la emergencia en $2.8 \pm 1.3$ folículos (rango de 1 a 8 ). Lo anterior corrobora el reporte de Vaughan et al. (2004), quienes caracterizaron la onda folicular en alpacas en condiciones diferentes al de los Andes peruanos por el crecimiento de 1 a 3 folículos de 3 a $5 \mathrm{~mm}$ de una cohorte inicial de 8 a 10 folículos $<3 \mathrm{~mm}$. Por lo tanto, estos antecedentes confirman que las alpacas tienen un estrecho rango y menor número de folículos $\geq 3 \mathrm{~mm}$ durante el reclutamiento folicular; cifras que son menores al compararse con los dromedarios (12.8 \pm 0.9 folículos $\geq 3 \mathrm{~mm}$, rango $8-34, \mathrm{n}=17$ ) (Manjunatha et al., 2012) y bovinos (8 a 41 folículos de 3-4 mm) (Pierson y Ginther, 1987), una variabilidad al parecer, atribuida a las especies.

Webb et al. (2016) resaltan a la alimentación como parte de las influencias ambientales con efectos sobre el desarrollo folicular y ovocitario. Al respecto, San Martín y Van Saun (2014) mencionan que la alimentación con pastos naturales en el Perú es favorable de enero a abril (estación lluviosa y calurosa), pero con bajo nivel de proteína entre mayo y julio, para proteínas y energía entre agosto y octubre o potencialmente deficiente para energía entre noviembre y diciembre. Poco se sabe sobre la importancia de la alimentación en los primeros meses de la gestación, en especial para empadres tardíos, comprometiendo la reproducción de las futuras crías, con probables efectos en desmedro del desarrollo y establecimiento de la reserva ovárica, coincidente con la escasez de alimentos. Los efectos de la alimentación sobre la futura reproducción de las crías fueron demostrados por Mossa et al. (2013), cuando una restricción alimenticia en el primer trimestre de preñez en vacas redujo hasta en $60 \%$ el número de folículos antrales en los ovarios de los fetos a la evaluación posparto. Una buena reserva de folículos en el ovario se ve reflejada con una adecuada respuesta en la reproducción, fertilidad y tasas de preñez (Silva-Santos et al., 2013). 
Cuadro 2. Número de folículos reclutados $\geq 3 \mathrm{~mm}$ de diámetro durante tres ondas foliculares consecutivas en alpacas, según el grupo experimental ${ }^{1}$

\begin{tabular}{lllllllllllll}
\hline \multirow{2}{*}{$\begin{array}{c}\text { Onda } \\
\text { folicular }\end{array}$} & \multicolumn{3}{c}{ Grupo Exp. 01 } & \multicolumn{4}{c}{ Grupo Exp. 02 } & \multicolumn{3}{c}{ Total } \\
\cline { 2 - 11 } & $\mathrm{n}$ & Media \pm DE & Min. Máx. & $\mathrm{n}$ & Media \pm DE & Min. Máx. & $\mathrm{n}$ & Media \pm DE Min. Máx. \\
\hline Primera & 15 & $2.3^{\mathrm{a}} \pm 0.5$ & 2 & 3 & 11 & $5.1^{\mathrm{b}} \pm 1.8$ & 4 & 9 & 26 & $3.5 \pm 1.8$ & 2 & 9 \\
Segunda & 15 & $2.8^{\mathrm{a}} \pm 1.1$ & 1 & 4 & 11 & $3.5^{\mathrm{a}} \pm 1.4$ & 2 & 6 & 26 & $3.1 \pm 1.3$ & 1 & 6 \\
Tercera & 15 & $2.3^{\mathrm{a}} \pm 1.5$ & 1 & 6 & 11 & $3.1^{\mathrm{a}} \pm 1.6$ & 1 & 6 & 26 & $2.6 \pm 1.6$ & 1 & 6 \\
\hline${ }^{1}$ GE01: $\leq 3$ folículos emergentes con $\geq 3$ & mm de diámetro; GE02: $\geq 4$ folículos emergentes con \\
$\geq 3$ mm de diámetro \\
$\mathrm{n}=$ número de animales \\
Letras distintas en la misma fila indican diferencia significativa $(\mathrm{p}<0.05)$ a la prueba de \\
hipótesis de Mann-Whitney
\end{tabular}

En el Cuadro 2 se puede observar la poca sostenibilidad del número de folículos reclutados $\geq 3 \mathrm{~mm}$ en el GE02, los cuales se redujeron a similares cantidades al del GE01 en la segunda y tercera onda folicular. Esta reducción podría estar atribuida, entre otros factores, al estrés ocasionado por la manipulación interdiaria al examen ecográfico y por los días transcurridos hasta el inicio de la tercera onda.

Llama la atención la reducción del número de folículos reclutados entre la primera con las dos ondas foliculares siguientes. Vásquez (2018) reportó una alta repetibilidad $(\mathrm{r}=0.72)$ para el número de folículos reclutados $\geq 3 \mathrm{~mm}$ en alpacas en ondas foliculares sucesivas. Asimismo, Manjunatha et al (2012) lo reportan en camellos dromedarios $(\mathrm{r}=0.95)$ y Burns et al (2005) en bovinos $(\mathrm{r}=0.95)$. Este comportamiento podría estar influenciado por la superposición de las ondas foliculares, donde el tamaño y duración de la primera onda (Adams, 2001), aunado al efecto de la progesterona procedente del cuerpo lúteo producto de la inducción previa de un folículo preovulatorio pudieron influir en el inicio de la segunda onda folicular. Se conoce que mientras el folículo dominante (FD) no ovule o regresione se mantiene su efecto inhibitorio por las elevadas concentraciones de estradiol y liberación de FSH sobre otros folículos, así como por los efectos de la inhibina y andrógenos, mecanismos postulados para otras especies monovulares como el bovino (Aba, 2014). Por el contrario, cuando el FD inicia su regresión, se anula el efecto inhibitorio hacia el hipotálamo y se percibe inmediatamente la presencia de un folículo de mayor tamaño (futuro folículo dominante) acompañado de otros folículos subordinados de la nueva onda folicular (Vaughan et al., 2004), observándose una coexistencia entre el folículo en retroceso y el folículo en crecimiento, siendo difícil distinguirlos a la ecografía, fenómeno al que Aba (2014) denomina como ondas foliculares superpuestas.

La alta repetibilidad $(r=0.72)$ de folículos reclutados $\geq 3 \mathrm{~mm}$ encontrada por Vásquez (2018) fue estimada en ondas foliculares sucesivas no ovulatorias, iniciándose con la ovulación de un folículo preovulatorio $\geq 7 \mathrm{~mm}$. La presentación de 
ondas superpuestas en alpacas pudo afectar el número de folículos reclutados medibles por ultrasonografía; por lo que urge dilucidar la repetibilidad en ondas foliculares subsecuentes donde los folículos dominantes sean inducidos a la ovulación. De otro lado, el coeficiente de repetibilidad debe ser considerado con mucha cautela porque tiene un comportamiento muy variable (Harper, 1994), influido por el tamaño de las estructuras, el rango de variación de la medida y la precisión del instrumento de medición.

El inicio de las ondas foliculares depende de la regresión natural de los FDs, de allí que también afectan la duración de las interondas foliculares, siendo estas muy variable entre animales y de baja repetibilidad $(\mathrm{r}=$ 0.23 ). Vásquez (2018) indica un intervalo de $16.9 \pm 3.9$ días, pero se describen rangos entre 12 a 22 días (Vaughan et al., 2004). A longitudes de intervalo largas, mayor es el crecimiento del FD (Adams, 2001) y mayor es la secreción de $17 \beta$-estradiol (Chaves et al., 2002), con el subsecuente mayor efecto inhibitorio sobre el crecimiento folicular (Aba, 2014) y su desarrollo en la onda superpuesta.

Bravo et al. (1990) manifiestan que el surgimiento de una nueva onda folicular se da a los 2-3 días de haberse iniciado la regresión del FD en ondas foliculares no ovulatorias; lo que hace suponer la existencia de algún mecanismo que posibilite la emergencia folicular momentos previos o inmediatamente después de la regresión del FD. En otras especies, Aba (2014), indica que el reclutamiento folicular en ovejas necesita de niveles basales de gonadotropinas, mientras que el inicio del reclutamiento folicular en el bovino requiere de una oleada secundaria de FSH posterior al proceso ovulatorio, evidenciando una relación entre FSH y la aparición de la onda folicular; de otra parte, no se ha encontrado en llamas una correlación temporal entre la secreción de FSH y el desarrollo de folículos, por lo que se sugieren establecer métodos más sensibles para medir la FSH en estas especies (Aba, 2014).
Así mismo, aún no está claro el tamaño folicular a partir del cual los folículos se hacen sensibles a las gonadotropinas con las que las alpacas inician la onda folicular, tal y como está determinado para la especie porcina (1 mm), ovina (2 mm), bovina (3-5 $\mathrm{mm})$ y equina $(10 \mathrm{~mm})$ (Monniaux et al., 1997). Recientemente, Céliz (2017) en un estudio en alpacas $(n=35)$ determinó por histología el recuento de folículos antrales a partir de ovarios procedentes de matadero, resultando entre 6 y 32 folículos/ovario. Por tanto, falta determinar la sensibilidad de los folículos a las gonadotropinas para esclarecer el inicio de la onda folicular en alpacas y hacerse el seguimiento con transductores de poca profundidad, pero de mayor resolución al cual se incluyan folículos $\geq 2 \mathrm{~mm}$, detectables con transductor de $7.5 \mathrm{MHz}$ en alpacas (Pacheco et al., 2017). Una situación similar ocurrió en bovinos, donde la variabilidad de folículos emergentes y su repetibilidad no fueron detectadas hasta incluir folículos $<5$ o $6 \mathrm{~mm}$ de diámetro (Evans et al., 2011).

La presencia de folículos anovulatorios y folículos anovulatorios luteinizados en ambos grupos obedece a fallas de la ovulación, descritas por Rodríguez et al (2014) como una inadecuada liberación de LH, donde folículos grandes que iniciaron con su regresión y terminan luteinizándose o cuando folículos pequeños que aún no han adquirido su capacidad para responder a la LH terminan sin llegar a ovular. También se debe al uso de machos con baja concentración o potencia del factor inductor de la ovulación que no tendría acción sobre folículos entre 3 y 7 $\mathrm{mm}$. La presencia de folículos anovulatorios con el uso de eCG también fueron observados por Cervantes (2008) al usar 650 UI y Vivanco et al (2014a) con 1000 UI de eCG, atribuidos a la subunidad beta de su estructura molecular y alto contenido de ácido siálico, responsables de la vida media larga de la eCG (Schams et al., 1978). 
Cuadro 3. Alpacas con colección embrionaria y promedio de colección de embriones en alpacas superestimuladas con eCG, según el grupo experimental ${ }^{1}$

\begin{tabular}{|c|c|c|c|c|c|c|c|}
\hline \multirow[t]{2}{*}{ Grupos } & \multirow[t]{2}{*}{$\begin{array}{l}\text { Alpacas } \\
\text { (n) }\end{array}$} & \multicolumn{2}{|c|}{$\begin{array}{l}\text { Alpacas con } \\
\text { colección } \\
\text { embrionaria }\end{array}$} & \multirow[t]{2}{*}{ Media $\pm \mathrm{DE}$} & \multirow[t]{2}{*}{ Mínimo } & \multirow[t]{2}{*}{ Máximo } & \multirow[t]{2}{*}{$\begin{array}{l}\text { Total de } \\
\text { embriones }\end{array}$} \\
\hline & & $\mathrm{n}$ & $\%$ & & & & \\
\hline GE01 & 15 & $12^{\mathrm{a}}$ & 80.0 & $2.5^{\mathrm{a}} \pm 1.4$ & 1 & 5 & 30 \\
\hline GE02 & 10 & $7^{\mathrm{a}}$ & 70.0 & $2.3^{\mathrm{a}} \pm 1.1$ & 1 & 4 & 16 \\
\hline Total & 25 & 19 & 76.0 & $2.4 \pm 1.3$ & 1 & 5 & 46 \\
\hline
\end{tabular}

\section{Colección Embrionaria}

Una alpaca de GE02 tuvo que ser retirada del análisis de esta parte del estudio por presentar piometra. El número de alpacas con colección embrionaria, así como el promedio de embriones fue similar en ambos grupos (Cuadro 3). No se pudo colectar embriones en todas las alpacas superestimuladas, hecho que también fue reportado en un estudio retrospectivo por Vaughan et al (2013) al no colectarse ningún embrión en 33.5\% (548/1736) de alpacas estimuladas en Australia. Por otra parte, la cantidad de embriones recuperados fueron ligeramente superiores a lo obtenido por Huanca (2008) con $1.6 \pm 0.3$ embriones con 750 UI de eCG, pero inferior a los reportados por Cervantes (2008) con $4.3 \pm 0.39$ embriones con 650 UI de eCG, Vivanco (2013) con $3.52 \pm 3.01$ en el año 2010 y $4.18 \pm 2.28$ y $3.69 \pm 3.42$ en 2013 , utilizando 1000 UI de eCG. Al respecto, el número de embriones recuperados dependen del momento en que los embriones descienden hacia el útero (Sumar y Picha, 2014).
Entre los factores intrínsecos que pudieron afectar la tasa de recuperación embrionaria está la función de la fimbria durante la captación de los óvulos en un ovario superestimulado cuyo tamaño es mucho más grade de lo usual (Novoa et al., 1999; Taylor et al., 2001). Otros factores se asocian a la presencia de folículos grandes (Vivanco et al., 2014a), la luteinización folicular y alteraciones durante la ovulación debido a la vida media larga de la eCG descrita por Tibary et al. (2015). En menor grado, se puede mencionar el medio y la técnica de recuperación embrionaria (Huanca et al., 2006), ya que el sistema discontinuo empleado no siempre coincidió entre las cantidades infundidas y las recuperadas durante el lavado intrauterino.

La tasa de recuperación de embriones transferibles se muestra en el Cuadro 4. La calidad excelente y buena de los embriones (transferibles) está influenciada por factores externos como las técnicas y los medios usados en la colección (Huanca et al., 2006), 
Cuadro 4. Recuperación de embriones transferibles y no transferibles en alpacas superestimuladas con eCG, según los grupos experimentales ${ }^{1}$

\begin{tabular}{lccccccc}
\hline \multirow{2}{*}{ Grupos $^{1}$} & $\begin{array}{c}\text { Total } \\
\text { alpacas }\end{array}$ & \multicolumn{2}{c}{ Transferibles } & \multicolumn{2}{c}{ No transferibles ${ }^{1}$} & \multicolumn{2}{c}{ Total } \\
\cline { 3 - 8 } & $\mathrm{n}$ & $\%$ & $\mathrm{n}$ & $\%$ & $\mathrm{n}$ & $\%$ \\
\hline GE01 & 15 & $25^{\mathrm{a}}$ & 83.3 & $5^{\mathrm{a}}$ & 16.7 & 30 & 100.0 \\
GE02 & 10 & $12^{\mathrm{a}}$ & 75.0 & $4^{\mathrm{a}}$ & 25.0 & 16 & 100.0 \\
\hline Total & 25 & 37 & 80.4 & 9 & 19.6 & 46 & 100.0 \\
\hline
\end{tabular}

${ }^{1}$ GE01: $\leq 3$ folículos emergentes con $\geq 3 \mathrm{~mm}$ de diámetro; GE02: $\geq 4$ folículos emergentes con $\geq 3 \mathrm{~mm}$ de diámetro

Letras distintas en la misma columna indican diferencia $(p<0.05)$ a la prueba de chi cuadrado con corrección de continuidad.

${ }^{1}$ Incluye ovocitos no fertilizados

Cuadro 5. Embriones colectados según estadio de desarrollo en alpacas superestimuladas con eCG, según los grupos experimentales

\begin{tabular}{|c|c|c|c|c|c|c|c|c|}
\hline \multirow{3}{*}{ Grupos $^{1}$} & \multicolumn{6}{|c|}{ Desarrollo embrionario } & \multirow{2}{*}{\multicolumn{2}{|c|}{ Total }} \\
\hline & \multicolumn{2}{|c|}{ Blastocistos } & \multicolumn{2}{|c|}{ Mórulas } & \multicolumn{2}{|c|}{$\mathrm{ONF}^{1}$} & & \\
\hline & $\mathrm{n}$ & $\%$ & $\mathrm{n}$ & $\%$ & $\mathrm{n}$ & $\%$ & $\mathrm{n}$ & $\%$ \\
\hline GE01 & $28^{\mathrm{a}}$ & 90.3 & $2^{\mathrm{a}}$ & 6.5 & $1^{\mathrm{a}}$ & 3.2 & 31 & 100.0 \\
\hline GE02 & $13^{\mathrm{a}}$ & 76.5 & $3^{\mathrm{a}}$ & 17.6 & $1^{\mathrm{a}}$ & 5.9 & 17 & 100.0 \\
\hline Total & 41 & 85.4 & 5 & 10.4 & 2 & 4.2 & 48 & 100.0 \\
\hline
\end{tabular}

${ }^{1}$ GE01: $\leq 3$ folículos emergentes con $\geq 3 \mathrm{~mm}$ de diámetro; GE02: $\geq 4$ folículos emergentes con $\geq 3 \mathrm{~mm}$ de diámetro

Letras distintas en la misma columna indican diferencia $(p<0.05)$ a la prueba exacta de Fisher

${ }^{1}$ Ovocitos no fertilizados

calidad espermática, capacidad o experiencia del recolector y grado de pureza de la hormona superovulatoria, entre otros (Reyna, 2006). Asimismo, por factores internos de la alpaca asociados a la calidad de los ovocitos que forman parte de la onda folicular, consi- derando que la hiperestimulación ovárica perturba el desarrollo normal del proceso, dando lugar a ovocitos con calidad reducida (Krisher, 2004), tales como el reinicio precoz de la meiosis por la actividad $\mathrm{LH}$ de la eCG, aneuploidía y problemas en el desarrollo temprano del embrión (Monniaux et al., 1984). 
Los embriones colectados según grado de desarrollo se presentan en el Cuadro 5. Los resultados fueron mayores a $61 \%(11 / 18)$ de blastocistos reportados por Mendoza (2012). La presencia de mórulas podría explicarse por ovulaciones tardías (Reyna, 2006) que terminaron en fecundaciones tardías. El embrión, en su última transición por el oviducto en dirección al útero lo hace mayormente en etapa de blastocisto eclosionado (Bourke et al., 1991), probablemente función atribuida a la unión útero tubárica, sometiendo al embrión a un delicado proceso selectivo propia de la especie para retener embriones no competentes (Novoa et al., 1999).

Finalmente, la selección de alpacas según el RFA mediante un seguimiento ecográfico $(5 \mathrm{MHz})$ no representaría un criterio significativo a pesar de que en otras especies como el bovino, constituye un buen marcador del estado de la reserva ovárica y un buen predictor de la respuesta ovárica a la superestimulación (Monniaux et al., 2010; Rico et al., 2012), lo que corrobora la variabilidad intra o entre especies (Vivanco et al., 2014b). Los camélidos sudamericanos posean características fisiológicas propias y extremadamente variables e impredecibles a los programas superovulatorios (Sumar, 2013).

\section{Conclusiones}

- La diferencia significativa en el número de folículos ováricos $\geq 3 \mathrm{~mm}$ al inicio de la primera onda folicular en alpacas (GE01: $\leq 3$ folículos, GE02: $\geq 4$ folículos) presentó similar número y tamaño de folículos antrales al día 7.5, así como la cantidad y tamaño de cuerpos lúteos al día 14 como respuesta superestimulatoria a la aplicación de eCG.

- Se determinó cantidades similares de folículos anovulatorios y folículos luteinizados en ambos grupos experimentales.

- El número promedio de embriones colectados y embriones transferibles fueron similares entre los dos grupos experimentales.
- la diferencia significativa del número de folículos $\geq 3 \mathrm{~mm}$ al inicio de la primera onda folicular (GE01: $<3$ folículos, GE02: $\geq 4$ folículos) es insostenible en ondas foliculares sucesivas no ovula-torias, reduciéndose en el grupo GE02.

\section{Literatura Citada}

1. Aba M. 2014. Anatomy and physiology of reproduction in the female llama and alpaca. In: Cebra C, Anderson D, Tibary A, Van Saun R, Johnson L (eds). Llama and alpaca care. Canadá: Elsevier. p 140-150.

2. Adams G. 2001. Comparative aspects of follicular dynamics in camelids. Rev Inv Vet Perú 1: 142-147.

3. Adams GP, Sumar J, Ginther OJ. 1990. Effects of lactational status and reproductive status on ovarian follicular waves in llamas (Lama glama). J Reprod Fertil 90: 535-545. doi: doi:10.1530/jrf.0.0900535

4. Andrade J. 2007. Métodos de sincronización de la onda folicular en base a GnRH y LH y su efecto en la respuesta ovárica y tasa de preñez en alpacas y llamas. Tesis de Maestría. Lima, Perú: Univ. Nacional Mayor de San Marcos. 62 p.

5. Bourke DA, Adam CL, Kyle CE. 1991. Successful pregnancy following nonsurgical embryo transfer in llama. Vet Rec 128: 68. doi:10.1136/vr.128.3.68

6. Bravo PW, Fowler ME, Stabenfeldt GH, Lasley BL. 1990. Ovarian follicular dynamics in the llama. Biol Reprod 43: 579-585. doi: 10.1095/ biolreprod43.4.579

7. Burns DS, Jimenez-Krassel F, Ireland JL, Kmight PG, Ireland JJ. 2005. Numbers of antral follicles during follicular waves in cattle: evidence for high variation among animals, very high repeatability in individuals, and an inverse association with serum folliclestimulating hormone concentration. Biol Reprod 73: 54-62. doi: 10.1095/biolreprod.104.036277 
8. Céliz RH. 2017. Determinación de la reserva ovárica en alpacas mediante la concentración de hormona antimulleriana (AMH) en el plasma sanguíneo. Tesis de Maestría. Lima, Perú: Univ. Nacional Agraria La Molina.

9. Cervantes F, MP. 2008. Momento óptimo post cópula para la recuperación de embriones del útero de alpaca mediante método no quirúrgico. Tesis de Magíster. Lima, Perú: Univ. Nacional Mayor de San Marcos. 43 p.

10. Chaves MG, Aba M, Agüero A, Egey J, Berestin V, Rutter B. 2002. Ovarian follicular wave pattern and the effect of exogenous progesterone on follicular activity in non-mated llamas. Anim Reprod Sci 69: 37-46. doi:10.1016/s03784320(01)00173-7

11. Evans AC, Mossa F, Fair T, Lonergan P, Butler ST, Zielak-Steciwko AE, Smith GW, et al. 2011. Causes and consequences of the variation in the number of ovarian follicles in cattle. Soc Reprod Fertil 67: 421-429. doi: 10.7313/ upo9781907284991.032

12. Harper DGC. 1994. Some comments on the repeatability of measurements. Ringing \& Migration 15: 84-90. doi: 10.1080/03078698.1994.9674078

13. Huanca $W$. 2005. Aplicación de biotecnologías en el mejoramiento genético de llamas y alpacas. Fase 1: Desarrollo y evaluación de un protocolo. Lima, Perú: CSI Boletín 55. p 8-12.

14. Huanca T. 2008. Efecto de la administración de gonadotropinas exógenas (FSH y eCG) en la respuesta ovárica y la producción de embriones en alpacas (Vicugna pacos). Tesis Doctoral. España: Univ. Santiago de Compostela. 31 p.

15. Huanca W, Cárdenas O, Olazábal C, Ratto M, Adams G. 2001. Efecto hormonal y empadre sobre el intervalo a la ovulación en llamas. Rev Inv Vet Perú 1: 462-463.

16. Huanca W, Ratto $M$, Cordero A, Santiani A, Huanca T, Cárdenas $O$, Adams GP. 2006. Respuesta ovárica y transferencia de embriones en alpacas y llamas en la zona altoandina del Perú. En: V Congreso Mundial de Camélidos. Catamarca, Argentina.

17. Ireland JJ, Ward F, Jimenez-Krassel F, Ireland JLH, Smith GW, Lonergan $P$, Evans AC. 2007. Follicle numbers are highly repeatable within individual animals but are inversely correlated with FSH concentrations and the proportion of good-quality embryos after ovarian stimulation in cattle. Hum Reprod 22: 1687-1695. doi: 10.1093/humrep/dem071

18. Ireland JLH, Scheetz, D, JimenezKrassel F, Themmen APN, Ward F, Lonergan P, Smith GW, Perez GI, Evans ACO, Ireland JJ. 2008. Antral follicle count reliably predicts number of morphologically healthy oocytes and follicles in ovaries of young adult cattle. Biol Reprod, 79:1219-1225.

19. Jimenez-Krassel F, Folger JK, Ireland JL, Smith GW, Hou X, Davis JS, Lonergan P, Evans ACO Ireland JJ. 2009. Evidence that high variation in ovarian reserves of healthy young adults has a negative impact on the corpus luteum and endometrium during estrous cycles in cattle. Biol Reprod 80: 1272-1281. doi: 10.1095/biolreprod.108.075093

20. Krisher RL. 2004. The effect of oocyte quality on development. J Anim Sci 82: 14-23. doi: 10.2527/2004.8213_supplE14x

21. Mamani C, Huanca W, Echevarría L, Cordero A, Huanca WF, Limache T. 2016. Susceptibilidad del cuerpo lúteo a la prostaglandina $\mathrm{F}_{2 \alpha}$ en alpacas inducidas a ovulación con plasma seminal y GnRH. Rev Inv Vet Perú 27: 729-735. doi: 10.15381/rivep.v27i4.12558

22. Manjunatha BM, Pratap N, AlBulushi S, Hago BE. 2012. Characterization of ovarian follicular dynamics in dromedary camels (Camelus dromedarius). Theriogenology 78: 965973. doi: 10.1016/j.theriogenology.2012.05.011 
23. Mendoza VR. 2012. Producción y evaluación de embriones por superovulación mediante la utilización de la eCG y FSH en alpacas Huacaya (Vicugna pacos) a $2730 \mathrm{msnm}$. Tesis de Médico Veterinario. Ayacucho, Perú: Univ. Nacional de San Cristóbal de Huamanga. 80 p.

24. Monniaux D, Barbey S, Rico C, Fabre S, Gallard Y, Larroque H. 2010. AntiMüllerian hormone: a predictive marker of embryo production in cattle? Reprod Fert Develop 22: 1083-1091. doi: 10.1071/RD09279

25. Monniaux D, Mariana JC, Gibson WR. 1984. Action of PMSG on follicular populations in the heifer. J Reprod Fertil 70: 243-253. doi: 10.1530/jrf.0.0700243

26. Monniaux D, Huet C, Besnard N, Clément F, Bosc M, Pisselet C, Monget P. 1997. Follicular growth and ovarian dynamics in mammals. J Reprod Fertil 51:3-23.

27. Mossa F, Carter F, Walsh SW, Kenny $D A$, Smith GW, Ireland JL, Hildebrant TB, et al. 2013. Maternal undernutrition in cows impairs ovarian and cardiovascular systems in their offspring. Biol Reprod 88: 92. doi: 10.1095/biolreprod.112.107235

28. Novoa C, Franco E, García W, Pezo D. 1999. Dosis de gonadotropinas (eCG y hCG), superovulación y obtención de embriones en alpacas. Rev Inv Vet Perú 10: 48-53. doi: 10.15381/rivep.v10i1.6616

29. Novoa C, Sumar J. 1968. Colección de huevos in vivo y ensayo de transferencia en alpaca. En: Bol Extraordinario IVITA. p 31-34.

30. Pacheco J, Pezo D, Velez, V, Bravo W. 2017. Descripción ecográfica del inicio de la actividad ovárica en Alpacas (Vicugna pacos). Rev Inv Altoandinas 19: 195-200.

31. Pierson RA, Ginther OJ. 1987. Reliability of diagnostic ultrasonography for identification and measurement of follicles and detecting the corpus luteum in heifers. Theriogenology 28: 929-936. doi: 10.1016/0093-691X(87)90043-4
32. Reyna JL. 2006. Embryo transfer in south american camelids. Camelid Quarterly 5: 79-93.

33. Rico C, Drouilhet L, Salvetti P, Dalbiès-Tran R, Jarrier P, Touzé JL, Pillet E, et al. 2012. Determination of anti-Müllerian hormone concentrations in blood as a tool to select Holstein donor cows for embryo production: from the laboratory to the farm. Reprod Fert Develop 24: 932- 944. doi: 10.1071/ RD11290

34. Rodriguez J, Pearson L \& Tibary. 2014. Infertility and subfertility in the female camelid. In: Cebra C, Anderson D, Tibary A. et al. (eds). Llama and alpaca care. Canada: Elsevier. p 216-242.

35. Rosales AM, Sánchez A, Gutiérrez C. 2012. Follicular development in domestic ruminants. Trop Subtrop Agroecosystems 15: S147-S160.

36. San Martín F, Van Saun RJ. 2014. Feeding management systems. In: Cebra C, Anderson D, Tibary A, Van Saun R, Johnson L (eds). Llama and alpaca care. Canada: Elsevier. p 91-100.

37. Schams D, Menzer C, Schallenberger E, Hoffman B, Hahn J, Hahn R. 1978. Some studies of the pregnant mare serum gonatrophin (PMSG) and on endocrine responses after application for superovulation in cattle. In: Sreenan (ed). Control of reproduction in the cow. The Hague: Martin Nijhoff. p 122-142.

38. Silva-Santos KC, Marinho LSR, Santos GMG, Machado FZ, Gonzalez SM, Lisboa LA, Seneda M. 2013. Ovarian follicle reserve: emerging concepts and applications. Anim Reprod 10: 180-186.

39. Singh J, Dominguez M, Jaiswal R, Adams GP. 2004. A simple ultrasound test to predict the superstimulatory response in cattle. Theriogenology 62 : 227-243. doi: 10.1016/j.theriogenology.2003.09.020

40. Sumar J, Picha Y. 2014. Embryo transfer. In: Cebra C, Anderson D, Tibary A, Van Saun R, Johnson L (eds). Llama and alpaca care. Canada: Elsevier. p 315-321. 
41. Sumar JB. 2013. Embryo transfer in domestic South American camelids. Anim Reprod Sci 136: 170-177. doi: 10.1016/ j.anireprosci.2012.10.029

42. Taylor S, Taylor PJ, James AN, Denniston RS, Godke R. 2001. Alpaca offspring born after cross species embryo transfer to llama recipients. Theriogenology 53 1-344.

43. Tibary A, Pearson LK, Campbell A. 2015. Embryo transfer in camelids. Spermova 5: 234-252

44. Van Saun RJ, Herdt TH. 2014. Nutritional assessment. In: Cebra C, Anderson D, Tibary A, Van Saun R, Johnson L (eds). Llama and alpaca care. Canada: Elsevier. p 100-123.

45. Vásquez YA. 2018. Caracterización de la emergencia y repetibilidad de la onda folicular en alpacas (Vicugna pacos). Tesis de Médico Veterinario. Lima, Perú: Univ. Nacional Mayor de San Marcos. 62 p.

46. Vaughan JL, Macmillan KL, D'Occhio MJ. 2004. Ovarian follicular wave characteristics in alpacas. Anim Reprod Sci 80: 353-361. doi: 10.1016/ j.anireprosci.2003.08.002

47. Vaughan JL, Mihm M, Wittek T. 2013. Factors influencing embryo transfer success in alpacas - A retrospective study. Anim Reprod Sci 136:194- 204.
48. Vivanco WH. 2013. Strategies for superovulation, embryo production and transfer in sheep and alpacas. 29 $9^{\text {th }}$ Annual Meeting of the European Embryo Transfer Association (AETE), Turkey.

49. Vivanco MHW, Salazar MP, Gonzales MM, Tapia MA. 2014a. Comparative efficiency of gonadotropin-releasing hormone and luteinizing hormone in the induction of ovulation in superovulated alpacas. Reprod Fert Develop 26: 168168. doi: 10.1071/RDv26n1 Ab108

50. Vivanco W, Ponce D, Miguel M, Youngs $C$, Huamán E, León S, Asparrin M. 2014b. Repetibilidad de la respuesta ovárica y de la producción embrionaria en alpacas Huacaya superovuladas. Spermova 4: 77-79.

51. Webb R, Buratini J, HernandezMedrano JH, Gutierrez CG, Campbell BK. 2016. Follicle development and selection: past, present and future. Anim Reprod 13: 234-249. doi: 10.21451/19843143-AR883

52. Webb R, Campbell BK. 2007. Development of the dominant follicle: mechanisms of selection and maintenance of oocyte quality. Soc Reprod Fertil (Suppl)64: 141-163. doi: 10.5661/rdr-vi-141 